\title{
Research Review on Marine Search and Rescue
}

\author{
CHEN Xinqiang ${ }^{1}$, SHI Chaojian ${ }^{1}$, WANG Shengzheng ${ }^{1}$, WU Huafeng ${ }^{1}, X$ Tie $^{1}$ and KE Ruimin ${ }^{2}$ \\ 1. Merchant Marine College, Shanghai Maritime University, Shanghai 201306, China \\ 2. Department of Civil and Environmental Engineering, University of Washington, Seattle 98195, United States
}

\begin{abstract}
Locating the marine target in a quick and precise way is the crucial point of implementing SAR (search and rescue) at sea, which involves aspects of developing SAR strategy and detects the marine targets. As the effect of marine target detection restricts the SAR result directly, the study has focused on reviewing the previous research about marine target detection, especially dim marine target detection. What's more, small target detection under complex sea status is one of the severe challenges which is research's hotspot and needs more endeavor. Current research results and future research directions are discussed in the paper. The findings can provide systematic view of implementing maritime search and rescue for field researchers and governors.
\end{abstract}

Key words: Maritime accident, search and rescue, complex marine condition, dim target detection, smart ship.

\section{Introduction}

The process of economic globalization has sharply promoted the development of marine freight transport and tourism, which mounts marine accident as well. As the complexity of ocean atmosphere and limited tolerance of human physical to the ocean, the SAR (search and rescue) process should be finished as soon as possible. Employing the IT technologies such as computer vision and image processing to handle the SAR process is one of most efficient methodologies [1]. Villainous weather, surging of ocean waves, reflectance of sunlight from sea surface and some other complex marine conditions really impede the SAR process.

In fact, researchers, ship officials, and maritime regulators have developed several types of work flows to optimize searching efficiency. It is common sense that wise and reasonable SAR strategy reduces casualties and properties loss. However, it is not an easy task to organize the limited SAR resources to find the drowning personnel. One of the common senses is that determining appropriate searching route is the first

Corresponding author: WANG Shengzheng, Ph.D., professor, research fields: computer vision, data mining, machine learning, and video surveillance. step with less influence from SAR surroundings [2-7]. But, dividing and distributing the SAR personnel and equipment into several groups to find the targets is another obstacle. In addition, searching targets are dim to be found by our naked eyes and computers. Such problem really challenges the way of SAR. Consequently, target detection, especially the small target detection in the heavy marine conditions, has become the hotspot and difficulty in the navigation field.

\section{Search Methods and Strategies in Maritime Accident}

The outcome of SAR is intimately with the search strategy and technology means used in target detection. Hence experts majoring in marine SAR are exploring the approaches excellent in detection speed and accuracy.

\subsection{SAR Strategy}

CHEN et al. [8] analyzed the merits and demerits of domestic SAR equipment, focusing on the advantages and disadvantages of visible light, infrared and radar in SAR. They built maritime accident SAR decision system which took maximum accuracy of target detection. The decision system made proposals for 
SAR based on the different marine condition and weather. Similarly, HE [9] designed marine SAR decision system based on the sufficient analysis of the interior marine SAR decision process. She concretely illustrated the system in the aspects of system function, structure and assessment of SAR scheme.

LI [10] indicated the existing maritime SAR decision systems were primarily based on the digital vessel model which was carried out depends on the conduct of experts on the scene, lowering the SAR efficiency. Thus, LI proposed novel marine SAR decision system based on multi-agent semi-automatic marine SAR decision system. What's more, taking the characteristics of heterogeneity of multi-agent and shared features of ontology, LI improved semi-automatic marine SAR system integrating the technologies of multi-agent and ontology.

CHEN [11] probed into improving the SAR efficiency in terms of identifying SAR region and arrangement of SAR services. CHEN modeled the SAR region with mathematical statistics method and produced the specific cases for fixing the SAR region. After that, CHEN developed some theoretical optimum SAR algorithm, with the cubic found exponential model, which could assign SAR equipment and personnel in a state of optimization in the SAR procedure.

WU [12] made use of POM (princeton ocean model) model to the law of tide of eight tidal constituent and their movement to build forecast model of surface tide in east China sea. To simplify the research, WU only took the tide and permanent current into consideration, and succeeded in tracking the floating object on ocean surface with constructed tide forecast model and estimating the circulation \& climate ocean circulation. The method could reduce the range of SAR and improve rescue efficiency during sea accident.

\subsection{Marine Target Detection}

Marine rescue effect mainly depends on marine target detection and identification capability which leads more and more attention to the research of marine target detection.

CHEN and RAN [1] proposed some marine target detection algorithm based on image processing and decision tree. The algorithm extracted multi-image features of SAR targets which involved in hue, luminance, square, rectangularity and circularity. Then a feature tree with information gain was constructed employing the extracted five features. CHEN and RAN took advantage of the proposed algorithm to detect marine target in video sequences. The simulation result revealed the very approach could track and detect the marine target with higher efficiency and accuracy in comparison with traditional ones.

CHEN et al. [13] did some researches in marine target detection with the FFT (Fractional Fourier Transform) of sea clutter. They stated that values of sea clutter based on FFT followed Gaussian distributions and possessed self-similarity in statistical property. Consequently, it was reasonable to employ fractal theory to handle over the values of sea clutter. CHEN et al. provided the measure of feature distil and scale-interval-pick with fractal parameters and marine mobile target detection algorithm. The algorithm utilized statistics of slope distance fitting from fractal dimensions and curves.

HU et al. [14] made use of fluctuation analysis, non-fluctuation analysis and fractal scale analysis with wavelet to do some research about characteristics of sea clutter. The research mainly concentrated on the data about sea clutter amplitude, which was upon actual measurement, integration of amplitude and difference of amplitude. Their result showed sea clutter possessed fractal character in time scale, implicating time was the most significant factor in the analysis of sea clutter fractal character. Besides, the method of fluctuation analysis could detect target submerged in sea clutter and approach of non-fluctuation analysis could offer some helpful information about target.

Meanwhile, HU et al. [15] proposed construction function with multi-fractal theory to detect marine 
target drowed by sea clutter. The function fulfilled assignment of target detection making the best of fractal character of sea clutter. Furthermore, the research displayed that sea clutter had weak dynamic nature ranging from 0.01 second to several seconds. Such weak dynamic nature made the imitative effect in Weibull distribution, logarithm function and compound Gaussian function missed the initial expectation. At last, HU et al. [16, 17] found Reese tower distribution could fit difference of sea clutter sequence in dipping into the data of the actual measured 280 sea clutter time series.

To improve SAR, with technology of radar, efficiency and accuracy in some terrible weather condition and lower visibility circumstances, LU et al. [18] proposed some algorithm to determine the threshold of target with background of sea clutter in maximum likelihood estimation. The algorithm made use of available target information such as number of AIS (Automatic Identification System), length, location, as training sample to fix the scope of target area. The research as well displaying the method based on maximum likelihood estimation could offer better accuracy and efficiency with traditional constant false alarm rate method.

REN et al. [19] provided marine target detection based on the decomposition of singular solution which overcame the adverse effects of sea clutter in target detection. First of all, the method picked up color and brightness of marine circumstance images which embodied targets. Then singular value decomposition was conducted, according to the extracted two kinds of characteristics, to acquire principal components of different features. Moreover, rough saliency map was calculated from subtracting the principal components with initial features. Finally, spatial domain filter method was employed to remove sea clutter so as to make the target more salient.

XIAO et al. [20] had proposed iterative-merge-expansion method on account of similarity of texture feature to detect vessel target in heavy background of sea and land. The method adopted different texture features of waters and lands to distinguish waters and lands. On that basis, some local target background contrast algorithm applying morphology filtering was presented to identify marine vessel target.

M. Y. YANG and W. P. YANG [21] put forward some automatic vessel detection methods which were able to detect ship from complicated background of sea and sky. The automatic method boosted the signal to noise ratio by filtering some noises and clutter of marine images with the help of wavelet transform. Then, the sea-sky-line detection algorithm, on the basis of segmenting mean value of gradient strength was proposed to avoid vessel false detection and leak detection in the infrared image. Such step decreased the substantial region which involved the target. Hence, some-adapting threshold algorithm with row-column mean value, focusing on the feature matching, was raised to detect the vessel. Similarly, WEN et al. [22] presented multi-scale method of orthogonal wavelet to separate sea-sky-line from vessel so as to achieve the aim of target detection with heavy clutter background.

WU and LI [23] considered traditional time-difference method, detecting marine mobile target, could not suppress ocean surface of wave noises, they proposed method of quick marine mobile target detection in visible light sequences. The first step was constructing pyramid structure for all single frames images, filtering noises and clutter of frames by iterative inverted triangle module. After that, the improved time-difference method was quoted to detect marine target. According to different IR imaging features of wave and different objects, XIE et al. [24] employed Sobel operator to segment and detect the likeliest area for target detection.

YANG et al. [25] deemed that self-adapted Butterworth high-passing filter could detect small target around heavy sea-sky background in infrared images validly with drawbacks of high false alarm rate. They surmised that different frames in sequence may 
lead to energy variety of small targets due to different cut-off frequency. To solve the problem, YANG et al. presented self-adapted energy threshold in weak target region which could resolve defects of target sudden disappearance and abrupt change of the background.

\section{Small Target Detection in Marine Circumstance}

For the sake of ruling out interference of sea clutter from target detection, REN et al. [26] held some global and local saliency marine target inspection. The method took image sub-block and domain contrast as local saliency, obtaining global contrast from frequency domain tuning. Then local saliency and global saliency were normalized together. The final global saliency was acquired by the product of normalized local saliency and global saliency. The new global saliency could reserve weak target information which was vital in marine small target detection. Fig. 1 is the detection effect.

As the further research of REN et al. [26], they depicted some new marine target detection methods with visible light as well [27]. The method improved the saliency representation which was on the basis of frequency tuning. In another word, three feature

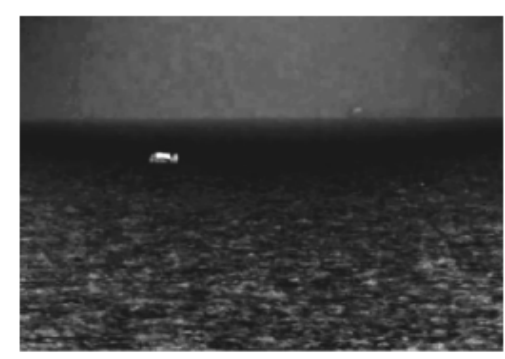

(a) global saliency

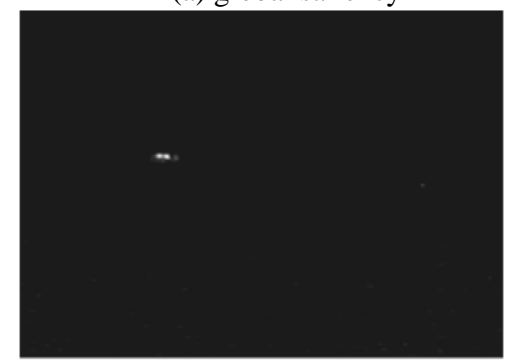

(c) new global saliency components of LAR space were applied with frequency domain tuning detection method merged as the new global saliency feature.

Sandeep et al. [28] presented marine target detection method with features online, sub-space and sea-clutter-suppressed. Compound Gaussian model was used to construct generalized-likelihood-quantitative measurement operator which could get marine image texture through assessing maximum value of expectation by the operator, which results in noise filtering and bettering the detect accuracy.

SHI et al. [29] pointed out retina of human eyes was relatively narrow. That's to say, the visible angle of human eyes may be no larger than 1 degree in both vertical and horizontal axis. Anything that is beyond the angle could not be identified correctly. What's more, human eyes were not good at focusing and light-brightness-adapting. The worst thing was that weak area in human eyes could be padded automatically. The awkward consequence was that human eyes would take small target as background, if the target was drowned by noises and background. To tackle such obstacle, SHI developed marine target

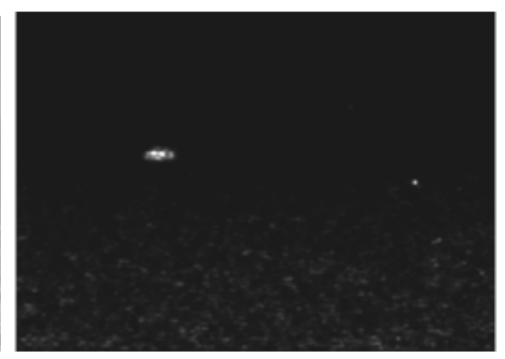

(b) local saliency

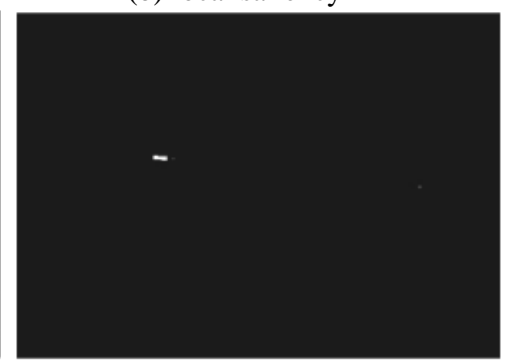

(d) saliency target

Fig. 1 Result of marine weak target detection based on global and local saliency. 
detection and visual enhancement system which blended with visible light, infrared, radar, satellite information and other available data.

WEN et al. [30] employed infrared technology to detect weak target in heavy clutter background. WEN et al. tried to rule out noise and strengthen dim target intensity with difference of mean value of each row in the image. Then top-hat filter was used to suppress the huge area of sea clutter and cloud cluster with appropriate rectangle structure element filtering the highlight target. Then local maximum value was obtained so as to fix the threshold value and segment the target. The effect of method mainly depends on structure element which means some improper structure element may lead to failure in the assignment. To overcome the problem, WEN et al. [31] made use of self-adapted wiener filtering approach to restrain the noises of clutter and Gaussian. And otus threshold was employed to discriminate small vessel target from sea-sky-line. Then top-hat operator was obtained to make dim vessel target more salient.

WEI et al. [32] proposed an algorithm to estimate the maximum sea-sky-line based on wavelet multi-resolution. Then a novel sea-clutter-suppressed method was presented in combination of wavelet and energy which could make potential vessel target area to a minimum level and accelerate search speed. WU et al. [33] proposed method of merging wavelet transform and multi-direction Gabor filtering. The method distinguished sea-sky-line and wave based on marine image characteristics. The Gabor wavelet was used to enhance feature of marine weak target so as to perfect detection ability.

Saeed et al. [34] proposed one supervised clustering method based on particle swarm algorithm and fuzzy C-means to identify target on the ocean. The method took full account of different feature vectors in RGB color space to discern the target. The algorithm utilized $3 \times 3$ transform matrix to transform RGB color space to object-oriented color space. Then particle swarm optimization algorithm was adopted to distinguish object color space and background color space. That is to say, particle swarm optimization was used to get minimum value of C-means clustering function during first and second transform process so as to identify marine weak target automatically.

CUI et al. [35] introduced dynamic planning into TBD (Track Before Detect) algorithm based on Hough transform to extract possible routes of weak target. And Kalman filter was imported in to filter the background of the extracted track and estimating object velocity.

Herselman et al. [36] pointed out operator performance excised great influence to ALQ (adaptive linear quadratic), particularly for those sea clutter in heterogeneous spectrum. Thus Herselman proposed self-adapted threshold mechanism to improve the poor performance of anti-interference of ALQ. Wensink [37] presented parameterized, real-time processing radar wave packet method which could detect and exclude sea-clutter information of radar wave packet successively, hence retain the target data.

XU [38] did some research about detecting method and technology for mobile vessel target, especially those weak mobile vessel targets, in infrared image. She used NARX network to predict images of remote marine scene, and acquired highlight-sea-object by the difference of the predicting image and actual infrared image. At the same time, she presented frame relevant target detection method in consideration of background of heavy sea clutter. The frame-relevant method got similar difference image with neighbor frames. The further research was that XU and FENG [39] proposed some weak target detections based on sea and sky background. The very algorithm combined background predicting and top-hat transform so as to filter wave noises.

ZUO et al. [40] did one ocean dim target method which was independent from prior information of ocean-surface. The approach sampled and smoothed the actual radar echo data with consideration of continuity of the track of ocean target and random distribution of sea clutter's amplitude range. Then the 
smoothed image would be transformed by Hough transform which could distinguish moving dim target from sea clutter for different amplitude range.

GUAN et al. [41] studied multi-fractal associated spectra of sea clutter and analyzed the echo similarity, in terms of similarity function, between sea clutter and marine dim target. Then support vector machine was used to differentiate sea clutter and dim target by determining echo threshold which would overcome the high false alarm in traditional measurement.

Based on infrared characteristics of marine circumstance and dim target, LIN et al. [42] constructed experience model for infrared radiation information of marine background and dim target. ZHENG and ZHOU [43] attempted to increase detection effect of infrared image by suppressing the infrared background. SUN et al. [44] suppressed background clutter with wavelet filter and increased signal to noise ratio by Context model. To make the dim target clearly, Bayes classification algorithm was used to segment images.

WANG et al. [45] took advantage of RBF-CML to recur the dynamic spatial and temporal feature of sea clutter. The method could display space shift without prior information of background information. Another advantage of the RBF-CML is that the very approach could distinguish the echo of sea clutter and dim target such that diminished error by time-domain-based RBF target detection.

WANG et al. [46] tried to deal with the drawbacks of time-consuming target detection methodology with the process of high-pass filter in spatial domain and self-adapted filtering. Therefore, WANG proposed an improved Donoho-wavelet-transform threshold method which employed hard threshold function to process the image. The method avoided the demerits of detecting multi-frames in order to detect the dim target. ZONG et al. [47] proposed fusion algorithm of multi-magnitude and multi-gradient table so as to detect infrared weak target in the sea-sky background. The method could satisfy result in single frame with relatively high false alarm.

\section{Method Comparison between the Available Sea Small Target Detection}

Generally speaking, the approach of marine weak target can be divided into following categories: infrared image detection radar-based target detection, visible-light-based method, morphology-based method, wavelet-based method and the combination of several approaches. The method based on infrared image can offer higher gray value which results in stark contrast between background and target. The superiority has prompted so many experts to implement dim target detection with infrared technology [48].

Nevertheless, the methods based on infrared technology own some intrinsic shortcomings. Firstly, imaging infrared system connately has larger noises which would disturb the quality of the imaging system. Secondly, the available infrared marine rescue equipment involves military-equipment and civil equipment, the latter has limited imaging range which leads to terrible detection result in huge search scope.

The navigation field has done the research about marine weak target with radar technology for a long period. But radar image can hardly acquire echo of ocean weak target and the received echo involves a huge amount of the information of sea clutter as well. The existing mathematics method cannot simulate the feature and distribution of sea clutter which restricts effect of marine weak target detection.

The marine weak target detection method based on visible light employs computer vision technology to search for the dim target instead of human eyes. To date, the well-developed technologies such as artificial intelligence, computer vision, deep learning [49, 50] and some other relative subjects have advanced the SAR efficiency vastly. However, the computer-vision-based marine dim target detection has following barriers: (1) weak target occupies small scale of pixels, sometimes even less than 5 pixels, which leads to lower signal to noise ratio, (2) the dim target to 
be searched is submerged with heavy background which impedes the SAR accuracy, (3) the algorithm for dim target search should not be time-consuming because of a huge amount of videos to be searched and human physical limit when floating in the cold and salty water. This is a really important challenge that should be tacked in priority.

Other methods based on morphology and wavelet analysis combine with the aforementioned technologies, such as XU and FENG [39] make use of morphology to filter noises from infrared images and eliminate region of noises and background. After excluding noise area, dim target can be identified by the very infrared features. The methods of wavelet-based have similar process as the morphology-based methods.

\section{Further Research and Conclusions}

As search process of sea accidents is often carried out in some extreme weather conditions, the SAR with combination of each available technology will boost the accuracy and efficiency of marine dim target detection, which is the hotspot and obstacle in the field as well.

(1) Dim target detection in the state of complicated ocean condition, lower signal to noise ratio. Small target detection process may have severe interferences in the heavy sea background. For instance, wave and gale around the small target may totally violate the search process. One of possible measurements is to extract features of target completely and deeply. With the help of deep learning [51], features of background and target can be fetched effectively and signal to noise ratio can raise vastly which will be beneficial to the SAR.

(2) Small target detection with fusion of every usable information and data such as infrared, radar, visible light and so forth. Different technology has different merits and demerits, so the fusion of available technologies and theories can make up the disadvantages of counterparts. In other words, images collected by different technologies can be rinsed and filtered with the help of morphology and artificial intelligence, such that target can be extracted effectively.

(3) Small target detection with interdisciplinary. Theories of morphology, visual attention mechanism, fuzzy mathematics and theories from some other subjects will be introduced for small target detection. The research methods of relevant problems and their solutions can be referred, such as debris detect in outer space [52].

For the purpose of improving SAR efficiency, the paper expounds the methods and strategies from both macroscopic and microscopic aspects. From the perspective of SAR strategy, the formulation of the search process is described in detail. On the other hand, the specific technologies and algorithms are critical for the accuracy and speed of SAR. So the research status in SAR is elaborated in the paper, with future research direction as well.

\section{Acknowledgements}

The authors appreciate the project support from China Scholarship Council, and the National Natural Science Foundation of China (51579143, 51379121, 61304230), Shanghai Shuguang Plan Project (No:15SG44) and China Postdoctoral Foundation (No. 2015M581585).

\section{References}

[1] CHEN, P. P., and RAN, X. 2010. "Marine Rescue Target Detection Algorithm Based on Decision Tree.” Journal of Shanghai Maritime University 31 (3): 1-4.

[2] Siljander, M., Venäläinen, E., Goerlandt, F., and Pellikka, P. 2015. "GIS-Based Cost Distance Modelling to Support Strategic Maritime Search and Rescue Planning: A Feasibility Study.” Applied Geography 57: 54-70.

[3] Kasyk, L., and Pleskacz, K. 2015. “An Adaptation of an Algorithm of Search and Rescue Operations to Ship Manoeuvrability." the International Journal on Marine Navigation and Safety of Sea Transportation 9 (2): 265-8.

[4] LI, W., and LIU, W. 2016. "The Optimized Selection Methods of Marine Search and Rescue Ships.” In Proceedings of the International Conference on Service 
Systems and Service Management.

[5] Lee, S., and Morrison, J. R. 2015. "Decision Support Scheduling for Maritime Search and Rescue Planning with a System of UAVs and Fuel Service Stations." In Proceedings of the International Conference on Unmanned Aircraft Systems.

[6] Lockett, J. 2016. "Development and Application of a Methodology to Assess the Adequacy of Search and Rescue on the River Thames.” Safety \& Reliability 36: 8-18.

[7] Matos, A., Martins, A., Dias, A., and Ferreira, B. 2016. "Multiple Robot Operations for Maritime Search and Rescue in euRathlon 2015 Competition.” Prensented at Oceans 2016, 1-7.

[8] CHEN, J. B., SHI, C. J., and CHEN, T. T. et al. 2010. "Research of SAR Decision System in Sea Accident." Presented at the Sixth China International Salvage Forum, 19-22.

[9] HE, Y. X. 2008. "Research and Design on the SAR DSS." M.Sc. thesis, Dalian Maritime University.

[10] LI, R. X. 2010. "Research of Maritime Search and Rescue Decision Support System Based on Ontology and Multi-agent.” M.Sc. thesis, Dalian Maritime University.

[11] CHEN, M. D. 2007. "Research on Maritime Searching Techniques.” M.Sc. thesis, Dalian Maritime University.

[12] WU, Z. D. 2003. "Structure and Functions of the Salvage Assistant System.” Ocean University of China.

[13] CHEN, X. L., LIU, N. B., SONG, J., GUAN, J., and HE, Y. 2011. "Fractal Feature Discriminant of Sea Clutter in FRFT Domain and Moving Target Detection Algorithm.” Journal of Electronics \& Information Technology 33 (4): 823-30.

[14] HU, J., GAO, J. B., Posner, F. L., ZHENG, Y., and Tung, W. W. 2006. "Target Detection within Sea Clutter: A Comparative Study by Fractal Scaling Analyses.” Fractals 14: 187-204.

[15] HU, J., Tung, W. W., and GAO, J. 2006. "Detection of Low Observable Targets within Sea Clutter by Structure Function Based Multi-fractal Analysis.” IEEE Transactions on Antennas and Propagation 54 (1): 136-43.

[16] HU, J., Tung, W., and GAO, J. 2006. "Modeling Sea Clutter as a Non-stationary and Non-extensive Random Process.” In Proceedings of IEEE Conference on Radar, $1-5$.

[17] HU, J., Tung, W., and GAO, J. 2006. “A New Way to Model Non-stationary Sea Clutter.” IEEE Signal Processing Letters 16 (2): 129-32.

[18] LU, Y., YING, S. J., SUN, Z., SHI, C. J., and ZOU, X. P. 2012. "Target Detection Algorithm for Marine Radar Based on Maximum Likelihood Estimate.” Journal of Shanghai Maritime University 33 (4): 1-4.
[19] REN, L., SHI, C. J., and RAN, X. 2012. "Saliency Detection for Sea Visual Scene Using SVD.” Computer Engineering and Applications 48 (23): 161-4.

[20] XIAO, L. P., CAO, J., and GAO, X. Y. 2007. "Detection for Ship Targets in Complicated Background of Sea and Land.” Opto-Electronic Engineering 34 (6): 6-10.

[21] YANG, M. Y., and YANG, W. P. 2008. "Automatic Detection Method of IR Warship Target in the Complex Sea-Sky Background.” Infrared and Laser Engineering 37 (4): 638-41.

[22] WEN, P. Z., SHI, Z. L., and YU, H. B. 2003. "Wavelet Transform-Based Detection for Small IR Target in Complex Sea Background.” LASER \& INFRARED 33 (6): 449-52.

[23] WU, Q. Y., and LI, C. H. 2007. "Novel Method for Moving Maritime Objects Detection.” Computer Engineering and Applications 43 (14): 213-6.

[24] XIE, X. Z., HONG, J. X., and XIAO, S. X. 2009. "Effective Method for Moving Objects Detection on Sea Surface.” Computer Engineering and Applications 45 (4): 225-6, 231

[25] YANG, L., YANG, J., and ZHENG, Z. L. 2006. "Detecting Infrared Small Targets Based on Adaptive Local Energy Threshold under Sea-Sky Complex Backgrounds.” Journal of Infrared and Millimeter Waves 25 (1): 41-5.

[26] REN, L., SHI, C. J., and RAN, X. 2012. “Small Target Detection in Ocean Environment Using Local and Global Saliency.” Journal of Shanghai Maritime University 33 (2): 1-5.

[27] REN, L., SHI, C. J., and RAN, X. 2012. "Small Target Detection Method under Sea Surface Environment Using Revised Frequency Tuned Saliency Detection.” Journal of Images and Graphics 17 (3): 365-9.

[28] Sira, S. P., Cochran, D., Papandreou-Suppappola, A., Morrell, D., Moran, W., and Howard, S. 2006. “A Subspace-Based Approach to Sea Clutter Suppression for Improved Target Detection.” Presented at the Fortieth Asilomar Conference on Signals, Systems and Computers, 752-6.

[29] SHI, C. J., XU, K. Y., PENG, J., and REN, L. 2008. "Architecture of Vision Enhancement System for Maritime Search and Rescue.” In Proceedings of the 8th International Conference on ITS Telecommunications, 12-7.

[30] WEN, P. Z., SHI, Z. L., and YU, H. B. 2003. “A Detection Method for IR Point Target on Sea Background Based on Morphology.” Opto-Electronic Engineering 30 (6): 55-8.

[31] WEN, P. Z., SHI, Z. L., and YU, H. B. 2003. "Automatic Detection Method of IR Small Target in Complex Sea Background.” Infrared and Laser Engineering 32 (6): 590-3. 
[32] WEI, Y., SHI, Z. L., and YU, H. B. 2003. "Wavelet Analysis Based Detection Algorithm for Infrared Image Small Target in Background of Sea and Sky." In Proceedings of the 3rd International Symposium on Image and Signal Processing and Analysis, 23-8.

[33] WU, J. D., MAO, H. C., and ZHANG, T. X. 2007. "Knowledge Based Detection for Small Target in Rotation on Sea Background.” Infrared and Laser Engineering 36 (2): 222-5.

[34] Mirghasemi, S., Sadoghi Yazdi, H., and Lotfizad, M. 2012. “A Target-Based Color Space for Sea Target Detection." Applied Intelligence 36 (4): 960-78.

[35] CUI, L., QU, C. W., and HUANG, Y. 2007. "Dim Target Detection and Its Movement Information Extracting with Heavy Sea Clutter Background.” Journal of Naval Aeronautical Engineering Institute 22 (1): 137-40, 144.

[36] Herselman, P. L., and De Wind, H. J. 2008. "Improved Covariance Matrix Estimation in Spectrally Inhomogeneous Sea Clutter with Application to Adaptive Small Boat Detection.” In Proceedings of the International Conference on Radar, 94-9.

[37] Wensink, H. E. 2000. "On Parametric Detection of Small Targets in Sea Clutter.” In Proceedings of the Third International Conference on Information Fusion, 17-24.

[38] XU, K. Y. 2006. "Research of Mobile Vessel Detecting and Tracking Based on Infrared Images.” Ph.D. thesis, Shanghai Maritime University.

[39] XU, K. Y., and FENG, D. 2009. "A Method for Detecting Small Infrared Targets in the Sea or Sky.” Journal of Harbin Engineering University 30 (9): 996-1000.

[40] ZUO, L., LI, M., ZHANG, X. W., and LUO, X. Y. 2012. "Small-Target Detection in Sea Clutter Based on Improved Hough Transform.” Journal of Electronics \& Information Technology 34 (4): 923-8.

[41] GUAN, J., LIU, N. B., ZHANG, J., and SONG, J. 2010. "Multi-fractal Correlation Characteristic of Real Sea Clutter and Low-Observable Targets Detection.” Journal of Electronics \& Information Technology 32 (1): 54-61.
[42] LIN, Y. P., SHEN, Y. J., and XU, K. Y. 2009. "Research on Infrared Radiation Characteristics of Small Target in Complex Marine Environment and Image Simulation.” Infrared 30 (8): 36-40.

[43] PENG, J. X., and ZHOU, W. L. 1999. "Infrared Background Suppression for Segmenting and Detecting Small Target.” Acta Electronica Sinica 27 (12): 47-51, 8.

[44] SUN, G. D., JI, S. P., and ZHOU, Z. 2010. “Dim Small IR Sea Target Detection Based on Wavelet and Context Model.” Infrared Technology 32 (2): 97-100.

[45] WANG, F. Y., LU, Z. Z., YUAN, G. N., and ZHOU, W. D. 2009. "Detection of Small Targets within Sea Clutter Using Spatial Temporal Chaos." Chinese Journal of Scientific Instrument 30 (6): 1180-5.

[46] WANG, W. L., HANG, B. J., and ZHANG, H. P. 2009. "A New Algorithm for Infrared Small Target Detection in the Sea Clutter Background." Acta Photonica Sinica 38 (3): 725-8.

[47] ZONG, S. G., WANG, J. A., and CHEN, Q. S. 2005. "New Detection Algorithm for Dim IR Point Targets under Complicated Sea and Sky Background." Opto-Electronic Engineering 32 (4): 9-12.

[48] RAN, X., and REN, L. 2010. "Small and Weak Target Detection on Sea Surface Based on Visible Light Video Image Processing." Journal of Shanghai Maritime University 31 (2): 11-7.

[49] Längkvist, M., Karlsson, L., and Loutfi, A. 2014. “A Review of Unsupervised Feature Learning and Deep Learning for Time-Series Modeling.” Pattern Recognition Letters 42: 11-24.

[50] Schmidhuber, J. 2015. "Deep Learning in Neural Networks: An Overview.” Neural Networks 61: 85-117.

[51] WONG, W. K., and SUN, M. 2011. "Deep Learning Regularized Fisher Mappings." IEEE Transactions on Neural Networks 22 (10): 1668-75.

[52] Cho, S., Huh, S., Shim, D. H., and Choi, H. S. 2013. "Vision-Based Detection and Tracking of Airborne Obstacles in a Cluttered Environment." Journal of Intelligent \& Robot Systems 69 (1-4): 475-88. 